\title{
Chronic disease: a core construct in psychogeriatrics
}

Most trainees, whether social workers, physicians, psychologists or nurses, are attracted to their career choice by the desire and hope that they will "cure" people and restore them to full health. Cures do occur in all of these fields, of course, but it is a surprise to most people to learn that cures are the exception and that chronicity is the basis of the care needs for a majority of individuals in most settings.

This is particularly true in geriatrics, in which individuals with multiple, persistent conditions are the norm. In the mental health field, some disorders are recurrent, for example, major depression and bipolar disorder, some conditions are persistent or lifelong, for example, personality vulnerabilities that predispose to distress when a stressful event occurs, and others are progressive at the present time such as schizophrenia and most dementias.

Is this necessarily a negative? I do not believe so. While there are many individuals who can be cured by an antibiotic, brief psychotherapy, surgery, or referral to the correct agency or help through a short-term crisis, there are many more whose problems are persistent, partially responsive to intervention, or progressive. Cures can occur in psychogeriatrics: Patients with major depression can fully recover and never have another episode, the identification of an available social resource can permanently solve a vexing and upsetting problem, ventriculo-peritoneal (VP) shunts can cure normal pressure hydrocephalus (NPH). However, cures are not the norm. Caring for chronic disease is central to all of the caring professions, but particularly to psychogeriatrics, in part, because of the successes of the past century through improved sanitation, antibiotics, and social safety nets.

I believe that pscyhogeriatrics attracts care providers who embrace the notion that helping individuals and families with chronic problems is beneficial and important. Psychogeriatrics cuts across artificial barriers erected by specialty labels, as do the principles that underlie the care of people with chronic illness. The care of the chronically ill requires unique skills. Acknowledging this and identifying what these are will help design healthcare systems that better meet the needs of the chronically ill and better train individual practitioners to meet these needs.

Cures will continue to be developed; some cancers that were untreatable 30 years ago, such as testicular cancer, are now curable. Better maintenance therapies will also continue to be developed; drug treatments for HIV/AIDS have restored individuals with this chronic illness to fully active lives. Twenty years ago no such treatments for HIV/AIDS were available. Researchers in psychogeriatrics will and should remain in the forefront of seeking such treatment advances. At the same time, psychogeriatricians of all backgrounds must continue to advocate for better care of the chronically ill, for more research into the causes and treatments of chronic illness, and for better systems of care that target improved quality of life for those with chronic illness.

What are the goals of care of those with chronic illness? What are the skills needed to provide the best care? What are the characteristics of systems that deliver the best care? I believe these questions have not received adequate attention and that psychogeriatricians should play a central role in finding the answers. The goal is not to "defend" the field of pscyhogeriatrics, but rather to be part of the solution. The mental, physical, and social challenges faced by the elderly require the integrated care of individuals with expertise in many fields. This is one of the attractions of psychogeriatrics. Disorders of the brain and mind are so prevalent and play such a major role in the lives of older individuals and their carers that psychogeriatricians must be among the leaders and advocates for better chronic care models. At the same time, we should strive to better enumerate the characteristics of care at multiple levels: the individual practitioner, the local unit of care, the regional provider, and the designers of healthcare systems. The following is offered as a beginning effort to identify some general principles that underlie chronic illness care and to identify the skill sets needed by individuals and systems to deliver the highest quality care. These are derived from my experience and lack empirical support. They are offered in the spirit of stimulating discussion about a crucial issue affecting our future as practitioners and patients.

1. Be as specific as possible in enumerating the medical, psychosocial, and psychological/psychiatric impairments and diagnoses of individuals. The complexity of multiple chronic illnesses can be overwhelming. The benefit of identifying disorders and problems specifically is that it can frame specific interventions and targets of care. 
2. Identify both the abilities and vulnerabilities of the individual. These include functional abilities and impairments, intrapersonal strengths and weaknesses (personality traits, wisdom gained from experience), environmental supports, social assets and vulnerabilities (family, friends, finances), and cognitive capacities and impairments.

3. Make sure accurate information is readily available about both the individual person and the disorders and situations they face. Patients, family members, and care providers should have accurate information. Accurate information systems can help dispel inappropriate hopelessness and unrealistic optimism. Information should be in forms that are easily understood, easily accessible (written hard copy; web-based; culturally relevant), and available when the recipient needs it. Information often needs to be repeated. Methods for addressing misunderstandings and misperceptions need to be a part of any information system.

4. Recognize that the emotional adjustment to chronic illness is often different from adjustment to the sequelae of acute illness. Chronic illnesses often introduce new problems and increase in complexity over time. Grief, the set of emotions and experiences through which humans pass after a loss, follows a roughly linear path that is interrupted by ups and downs but usually leads to a resolution and acceptance. Chronic illness, because it does not resolve and is often progressive, can repeatedly reignite a grieving process that further adds to disablement. Having a range of resources to address this should be a part of any chronic care system.

5. Enhance remoralization. Human beings are resilient, even in the face of very difficult circumstances. The day-to-day care of people with chronic illnesses teaches us that many people not only adapt to chronic impairments but also find ways to continue living a meaningful, enjoyable, and active life. The assumption that the irreversible or progressive characteristics of chronic illness are insurmountable and permanently demoralizing is not borne out in the real world of chronically ill. Steps 1-4 above can contribute to adaptation, but resilience and adaptation to change are wellestablished characteristics of many individuals. Individual practitioners and systems need to recognize these innate strengths and capitalize upon them. They also need to recognize that some individuals and families are less able to adapt and need resources specific to their situation that can increase the likelihood that they will adjust over time.

6. Acknowledge that the complexity of multiple chronic conditions introduces unique challenges. New problems can be difficult to recognize. Disorders, medications, social issues, intrapersonal strengths and vulnerabilities, and cultural norms can interact in ways that are impossible to predict. Acknowledging that unexpected and unpredictable events can occur should lead to methods and mechanisms for earlier recognition and, hopefully, better interventions. Clinicians must be alert for changes and unexpected turns of events. Having consultants and systems available when unexplained, unexpected, or complex events occur has the potential to identify new issues earlier and interrupt their progression to chronicity or to minimize their effect on existing chronic conditions.

Past successes in developing cures for many acute conditions and preventions for many acute and chronic conditions make me optimistic that further advances will prevent many chronic illnesses from occurring (primary prevention) and will meaningfully mitigate the negative outcomes of others (secondary prevention). We already have available approaches that can improve the lives of people with chronic illnesses (tertiary prevention), but the trend of increasing prevalence of chronicity will undoubtedly continue. We should see this as an opportunity to relieve suffering and to improve quality of life, the two mainstays of health systems, even those that emphasize prevention. Psychogeriatrics should embrace its role in the care of the chronically ill and be among the leaders in improving care in the next 25 years.

\section{Conflict of interest}

None.

\section{Peter V. RABINS}

Department of Psychiatry and Behavioral Sciences, Johns Hopkins School of Medicine, Baltimore, Maryland, USA

Email: pvrabins@jhmi.edu 